\title{
Evaluation of Self-Produced Phantom Usafulness for image Quality Control of Radiation Generator for Diagnosis
}

\author{
Ye-Won Park ${ }^{1}$, Cheong-Hwan Lim*², Beom-Hee Han ${ }^{3}$ \\ ${ }^{1}$ Ph.D., Dept. Of Health Care, Hanseo University, 46, Hanseo 1-ro, Haemi-myeon, Seosan-si, \\ Chungcheongnam-do, 31962, Rep. of KOREA \\ ${ }^{2}$ Professor, Dept. Of Health Care, Hanseo University, 46, Hanseo 1-ro, Haemi-myeon, Seosan-si, \\ Chungcheongnam-do, 31962, Rep. of KOREA \\ ${ }^{3}$ President, Radiation Science Technology Laboratory, Asan-si, Chungcheongnam-do, 31561, Rep. of KOREA \\ srtpyw@nate.com¹, 1ch116@hanseo.ac.kr², raphael121215@hanmail.net \\ Corresponding author*: Cheong-Hwan Lim, E-mail : lch116@hanseo.ac.kr
}

Article History:Received:11 november 2020; Accepted: 27 December 2020; Published online: 05 April 2021 \begin{abstract}
Background/Objectives: Quality control can improve the quality of medical care along with the stability of diagnostic X-ray generator. Regular quality control provides reliable quality control of the machine and maintains consistency of general imaging using radiation for efficient diagnosis.

Methods/Statistical analysis: A phantom for quality control of diagnostic X-ray generator was produced using a 3D printer. Quantitative and qualitative evaluation of the phantom utility was conducted by modifying images acquired using S and D companies' tools with Source to Image-Receptor Distance (SID) levels ranging between $130 \mathrm{~cm}$ and $180 \mathrm{~cm}$. The evaluation indices were determined based on the analysis of field compliance, uniformity, low and high-contrast resolution, and linearity.

Findings: The evaluation was conducted by acquiring and changing the radiographic image to SIDs between $130 \mathrm{~cm}$ and $180 \mathrm{~cm}$ using the indigenous phantom. The field compliance of $\mathrm{S}$ and $\mathrm{D}$ companies in terms of quantitative evaluation indices was both appropriate within $\pm 1 \%$ according to the SID change. To ensure a uniform SID $130 \mathrm{~cm}$, the internal and external means of S company were 893 and 943, respectively, while those of the D company were 228.1 and 261.4, respectively. At an SID of $180 \mathrm{~cm}$, the internal and external means of the S company were 928.1 and 958.4 , respectively, while those of the D company were 257.2 and 299, respectively. A characteristic of the DR system was identified to ensure linearity, altered exposure dosage according to the step wedge height, and the difference in SI values according to the characteristics of the equipment and linearity. The qualitative evaluation indices were determined by identifying the size of the hole under high-contrast resolution up to $0.8 \mathrm{~mm}$ and the bar size up to $1.6 \mathrm{lp} / \mathrm{mm}$. The low contrast resolution was evaluated with a C-D pattern, and at SID $130 \mathrm{~cm}$, the $\mathrm{S}$ company scored 124.6 points and the D company 116 points, and at $180 \mathrm{~cm}$, the $\mathrm{S}$ company scored 111.4 and the D company 104.6 points.

Improvements/Applications: The utility of the homegrown phantom in quality control was confirmed for each index. The medical institutions are required to introduce quality control regulations for general image examination using radiation. It is helpful to efficiently manage old equipment and improve public health and medical care by linking with the health insurance fee.
\end{abstract}

Keywords: DR system, 3D Printer, Quality Control, Phantom, Image Evaluation

\section{Introduction}

The benefits of diagnostic imaging depend on appropriate diagnostic equipment, examination methods and image interpretation. Inappropriate imaging has an adverse effect on public health due to unnecessary radiation exposure and duplicate examination, along with the risk of misdiagnosis. Over-exposure to X-rays leads to degradation of image quality due to the wide and dynamic range of the diagnostic Digital Radiography (DR) system, and the convenience and user-friendly features of the equipment used[1,2]. Besides, it is necessary to renew the work habits and perceptions to minimize the irradiation and the exposure dose for patients. It is believed that continuous interest, regular education and inspection, and various educational opportunities to reduce exposure dose are necessary [3].In Korean medical institutions, only regular inspections for electrical and mechanical safety management of diagnostic radiation generators have been conducted, without quality control of images. Also, there are no institutionalized programs. Introduction of quality control of diagnostic radiation generator and its implementation is believed to contribute to enhanced image quality, reduced radiation exposure, and decreased national health expenditure through efficient use of equipment, leading to a positive impact on the promotion of national health[4].Currently in Korea, once the diagnostic X-ray generators are judged appropriate based on regular examination, no further examinations are conducted for 3 years. Therefore, the status of the equipment that is outside the reference value within three years is impossible to determine. The old diagnostic X-ray generators increase the exposure of patients or radiation workers, and the probability of misdiagnosis due to degraded image quality. Therefore, it is necessary to ensure reliability through quality control of medical images.

*Corresponding author: Cheong-Hwan Lim,

Professor, Dept. Of Health Care, Hanseo University, 46, Hanseo 1-ro, Haemi-myeon, Seosan-si,

Chungcheongnam-do, 31962, Rep.of KOREA.E-mail : lch116@hanseo.ac.kr 
Accordingly, this study developed an indigenous phantom appropriate for measurement of quality control indices for diagnostic X-ray generators and suggests ways to improve its utility.

\section{Materials and Methods}

\subsection{Research equipments}

In this study, as shown in Figure 1, the diagnostic X-ray generators equipped with the DR system were YSIO (SIEMENS, Germany) and RAD-14 (DongKang, Korea). Image acquisition was carried out and stored as a 16bit image using an indirect conversion method. The number of pixels for images generated by $\mathrm{S}$ company was $3,040 \times 3,040(144 \mu \mathrm{m})$ and those of D company, 3,072 ×3,072 (143 $\mu \mathrm{m})$. The detector size was $17 \times 17$ inches, and similar Scintillator (CsI) and semiconductor materials (a-Si) were used for the detector. The size of the homegrown phantom was $200 \times 200 \times 33 \mathrm{~mm}$ (width $\times$ length $\times$ height).

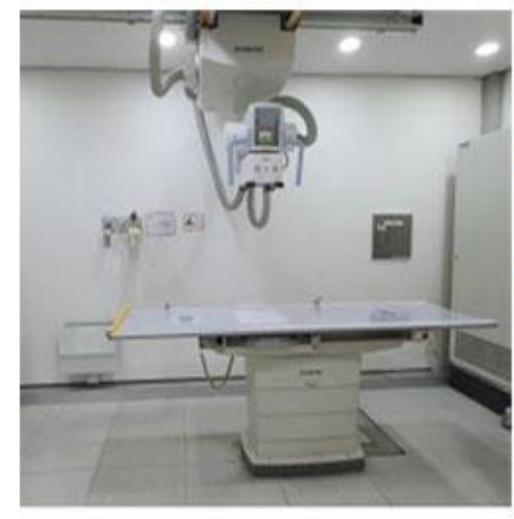

(a)

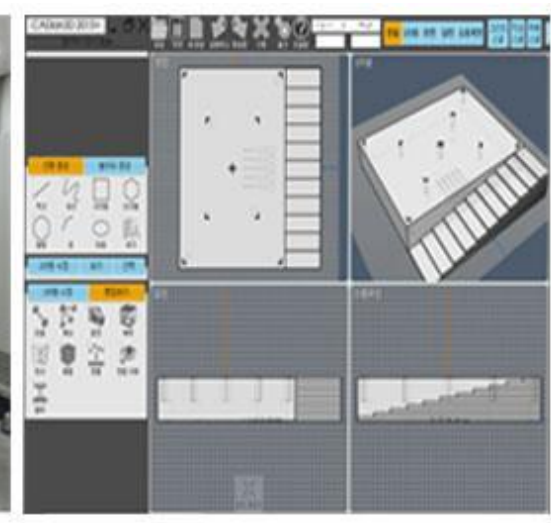

(b)

\subsection{Research method}

Figure 1. Research equipment

\subsubsection{Phantom image acquisition}

Image evaluation of phantom was based on the tube voltage $(\mathrm{kVp})$, tube current-time (mAs), and titer according to changes in the_Source to Image-Receptor Distance (SID). Phantom images were acquired at a SID of $130 \mathrm{~cm}$, and those of the $\mathrm{S}$ company were measured with a tube voltage of $50 \mathrm{kVp}$ and a tube current-time of $3 \mathrm{mAs}$, while those of $\mathrm{D}$ company were measured with a tube voltage of $50 \mathrm{kVp}$ and a tube current-time of 2 mAs. At SID $180 \mathrm{~cm}$, the phantom image acquired using the generator produced by $\mathrm{S}$ company was measured at a tube voltage of $50 \mathrm{kVp}$ and a tube current-time of $4 \mathrm{mAs}$, while that of $\mathrm{D}$ company was measured with a tube voltage of $50 \mathrm{kVp}$ and a tube current-time of $3 \mathrm{mAs}$ [Table 1].

Table 1. Phantom inspection conditions

\begin{tabular}{ccccc}
\hline manufacturer & \multicolumn{2}{c}{$130 \mathrm{~cm}$} \\
\hline S company & $50 \mathrm{kVp}$ & $3 \mathrm{mAs}$ & $50 \mathrm{kVp}$ & $4 \mathrm{mAs}$ \\
D company & $50 \mathrm{kVp}$ & $2 \mathrm{mAs}$ & $50 \mathrm{kVp}$ & $3 \mathrm{mAs}$ \\
\hline
\end{tabular}

\subsubsection{Utility evaluation of Phantom}

The acquired images were evaluated by five radiologists with two or more years of clinical experience. A total of four images were selected, and the best phantom images for analysis were acquired at an SID of $130 \mathrm{~cm}$ and $180 \mathrm{~cm}$ for each equipment manufacturer. The utility evaluation of the phantom entailed quantitative and qualitative analyses. Field compliance, uniformity, and linearity were measured via quantitative evaluation, whereas qualitative evaluation was used to analyze high- and low-contrast resolution.

\section{Results}

\subsection{Uniformity}

While the Regions of Interest (ROI) of images acquired according to changes in SID from $130 \mathrm{~cm}$ to $180 \mathrm{~cm}$ were visually similar, the signal intensity (SI) varied as shown in Table 2 . The SI mean values for images generated by the S Company ranged from 893.4 to 958.4, those of the D Company varied from 228.1 to 299.

Table 2. SI value according to changes in SID.

\begin{tabular}{|c|c|c|c|c|c|}
\hline manufacturer & SID & ROI & Area & Mean \pm SD & Mean \\
\hline
\end{tabular}


Ye-Won Park ${ }^{1}$, Cheong-Hwan Lim*2, Beom-Hee Han ${ }^{3}$

\begin{tabular}{|c|c|c|c|c|c|}
\hline \multirow{8}{*}{ S company } & \multirow{4}{*}{$130 \mathrm{~cm}$} & (1) & 31.3 & $917.4 \pm 30.8$ & \multirow{2}{*}{893.4} \\
\hline & & (2) & 31.3 & $869.3 \pm 28.6$ & \\
\hline & & (3) & 31.3 & $914.9 \pm 29.7$ & \multirow{2}{*}{943.0} \\
\hline & & (4) & 31.3 & $971.0 \pm 25.2$ & \\
\hline & \multirow{4}{*}{$180 \mathrm{~cm}$} & (1) & 31.3 & $967.7 \pm 0.5$ & \multirow{2}{*}{928.1} \\
\hline & & (2) & 31.3 & $888.4 \pm 33.7$ & \\
\hline & & (3) & 31.3 & $956.6 \pm 39.5$ & \multirow{2}{*}{958.4} \\
\hline & & (4) & 31.3 & $960.3 \pm 32.5$ & \\
\hline \multirow{8}{*}{ D company } & \multirow{4}{*}{$130 \mathrm{~cm}$} & (1) & 31.3 & $248.8 \pm 36.7$ & \multirow{2}{*}{228.1} \\
\hline & & (2) & 31.3 & $207.5 \pm 31.3$ & \\
\hline & & (3) & 31.3 & $293.0 \pm 46.5$ & \multirow{2}{*}{261.4} \\
\hline & & (4) & 31.3 & $229.8 \pm 36.2$ & \\
\hline & \multirow{4}{*}{$180 \mathrm{~cm}$} & (1) & 31.3 & $298.5 \pm 41.9$ & \multirow{2}{*}{257.2} \\
\hline & & (2) & 31.3 & $215.9 \pm 35.5$ & \\
\hline & & (3) & 31.3 & $239.9 \pm 36.7$ & \multirow{2}{*}{299.0} \\
\hline & & (4) & 31.3 & $358.2 \pm 50.3$ & \\
\hline
\end{tabular}

\subsection{Field compliance}

As shown in Table 3, the horizontal and vertical error rates of images acquired by S company at SID $130 \mathrm{~cm}$ were $0.12 \%$ and $0.46 \%$, respectively. The horizontal and vertical error rates in case of D company were measured at $0.58 \%$ and $0.64 \%$, respectively.

Table 3. Collimation consistency at SID of $130 \mathrm{~cm}$

\begin{tabular}{|c|c|c|c|c|}
\hline \multirow{2}{*}{$\begin{array}{l}\text { Characteristics } \\
\text { Manufacturers }\end{array}$} & \multicolumn{2}{|c|}{ Horizontal error rate } & \multicolumn{2}{|c|}{ Vertical error rate } \\
\hline & $e_{1+} e_{2}$ & Error rate & $e_{3}+e_{4}$ & Error rate \\
\hline S company & $0+0.14 \mathrm{~cm}$ & $0.12 \%$ & $0.22+0.33 \mathrm{~cm}$ & $0.46 \%$ \\
\hline Judgment & \multicolumn{2}{|c|}{ Appropriate } & \multicolumn{2}{|c|}{ Appropriate } \\
\hline D company & $0.51+0.19 \mathrm{~cm}$ & $0.58 \%$ & $0.16+0.61 \mathrm{~cm}$ & $0.64 \%$ \\
\hline Judgment & \multicolumn{2}{|c|}{ Appropriate } & \multicolumn{2}{|c|}{ Appropriate } \\
\hline
\end{tabular}

As shown in Table 4, at an SID of $180 \mathrm{~cm}$, the horizontal and vertical error rates of images produced by $\mathrm{S}$ company were $0.38 \%$ and $0.41 \%$, respectively. The horizontal and vertical error rates in case of D company were $0.77 \%$ and $0.44 \%$, respectively

Table 4. Collimation consistency at SID of $180 \mathrm{~cm}$

\begin{tabular}{ccccc}
\hline \multirow{2}{*}{ Characteristics } & \multicolumn{2}{c}{ Horizontal error rate } & \multicolumn{2}{c}{ Vertical error rate } \\
\cline { 2 - 5 } Manufacturers & \multicolumn{2}{c}{$e_{1+e_{2}}$} & Error rate & \multicolumn{2}{c}{$e_{3}+e_{4}$} & Error rate \\
\hline S company & $0.36+0.32 \mathrm{~cm}$ & $0.38 \%$ & $0.63+0.1 \mathrm{~cm}$ & $0.41 \%$ \\
\hline Judgment & \multicolumn{2}{c}{ Appropriate } & \multicolumn{2}{c}{ Appropriate } \\
\hline D company & $0.80+0.58 \mathrm{~cm}$ & $0.77 \%$ & $0.64+0.16 \mathrm{~cm}$ & $0.44 \%$ \\
\hline Judgment & \multicolumn{2}{c}{ Appropriate } & Appropriate \\
\hline
\end{tabular}

\subsection{Low contrast resolution}

Five radiologists with more than two years of experience visually evaluated and scored the images acquired for a C-D pattern using equipment manufactured by S and D companies based on SID changes. As shown in Table 5, at SID $130 \mathrm{~cm}$, the mean score of S company was 124.6, while that of D company scored 116 . At SID $180 \mathrm{~cm}$, the mean score of S company was 111.4, while that of D company was 104.6. 
Evaluation of Self-Produced Phantom Usafulness for image Quality Control of Radiation Generator for Diagnosis

Table 5. Low contrast resolution test

\begin{tabular}{ccccc}
\hline \multirow{2}{*}{ Classification } & \multicolumn{2}{c}{$130 \mathrm{~cm}$} & \multicolumn{2}{c}{$180 \mathrm{~cm}$} \\
\cline { 2 - 4 } & S company & D company & S company & D company \\
\hline Evaluator 1 & 125 & 108 & 101 & 90 \\
\hline Evaluator 2 & 149 & 108 & 116 & 108 \\
\hline Evaluator 3 & 132 & 123 & 131 & 93 \\
\hline Evaluator 4 & 109 & 116 & 93 & 116 \\
\hline Evaluator 5 & 108 & 125 & 116 & 104.6 \\
\hline Mean & 124.6 & 116 & 11.4 & 93 \\
\hline
\end{tabular}

\subsection{High contrast resolution}

The hole pattern was evaluated depending on whether or not the hole size and space can be identified according to the manufacturer of the diagnostic X-ray generator and the changes in SID. As shown in Table 6, at an SID of $130 \mathrm{~cm}$, the equipment manufactured both S and D companies was able to identify the hole pattern up to $0.8 \mathrm{~mm}$ in diameter, and at SID $180 \mathrm{~cm}$, both generators were able to identify the hole pattern up to $0.8 \mathrm{~mm}$ in diameter.

Table 6. Hole pattern test based on high-contrast resolution

\begin{tabular}{ccccc}
\hline \multirow{2}{*}{ Classification } & \multicolumn{2}{c}{$130 \mathrm{~cm}$} & \multicolumn{2}{c}{$180 \mathrm{~cm}$} \\
\cline { 2 - 4 } & S company & D company & S company & D company \\
\hline Evaluator 1 & $0.8 \mathrm{~mm}$ & $0.8 \mathrm{~mm}$ & $0.8 \mathrm{~mm}$ & $0.8 \mathrm{~mm}$ \\
\hline Evaluator 2 & $0.8 \mathrm{~mm}$ & $0.8 \mathrm{~mm}$ & $0.8 \mathrm{~mm}$ & $0.8 \mathrm{~mm}$ \\
\hline Evaluator 3 & $0.8 \mathrm{~mm}$ & $0.8 \mathrm{~mm}$ & $0.8 \mathrm{~mm}$ & $0.8 \mathrm{~mm}$ \\
\hline Evaluator 4 & $0.8 \mathrm{~mm}$ & $0.8 \mathrm{~mm}$ & $0.8 \mathrm{~mm}$ & $0.8 \mathrm{~mm}$ \\
\hline Evaluator 5 & $0.8 \mathrm{~mm}$ & $0.8 \mathrm{~mm}$ & $0.8 \mathrm{~mm}$ & $0.8 \mathrm{~mm}$ \\
\hline Mean & $0.8 \mathrm{~mm}$ & $0.8 \mathrm{~mm}$ & $0.8 \mathrm{~mm}$ & $0.8 \mathrm{~mm}$
\end{tabular}

The spacing of the hole pattern was identified up to $1.0 \mathrm{~mm}$, regardless of the SID change and manufacturer. The visual identification of the bar pattern was evaluated according to the manufacturer of the diagnostic X-ray generator and the changes in SID. As shown in Table 7, all bars were identified up to $1.6 \mathrm{LP} / \mathrm{mm}$, regardless of the SID change and manufacturer.

Table 7. Bar pattern test based on high-contrast resolution

\begin{tabular}{ccccc}
\hline \multirow{2}{*}{ Classification } & \multicolumn{2}{c}{$130 \mathrm{~cm}$} & \multicolumn{2}{c}{$180 \mathrm{~cm}$} \\
\cline { 2 - 4 } & S company & D company & S company & D company \\
\hline Evaluator 1 & $1.61 \mathrm{p} / \mathrm{mm}$ & $1.61 \mathrm{p} / \mathrm{mm}$ & $1.61 \mathrm{p} / \mathrm{mm}$ & $1.61 \mathrm{p} / \mathrm{mm}$ \\
\hline Evaluator 2 & $1.61 \mathrm{p} / \mathrm{mm}$ & $1.61 \mathrm{p} / \mathrm{mm}$ & $1.61 \mathrm{p} / \mathrm{mm}$ & $1.61 \mathrm{p} / \mathrm{mm}$ \\
\hline Evaluator 3 & $1.61 \mathrm{p} / \mathrm{mm}$ & $1.61 \mathrm{p} / \mathrm{mm}$ & $1.61 \mathrm{p} / \mathrm{mm}$ & $1.61 \mathrm{p} / \mathrm{mm}$ \\
\hline Evaluator 4 & $1.61 \mathrm{p} / \mathrm{mm}$ & $1.61 \mathrm{p} / \mathrm{mm}$ & $1.61 \mathrm{p} / \mathrm{mm}$ & $1.61 \mathrm{p} / \mathrm{mm}$ \\
\hline Evaluator 5 & $1.61 \mathrm{p} / \mathrm{mm}$ & $1.61 \mathrm{p} / \mathrm{mm}$ & $1.61 \mathrm{p} / \mathrm{mm}$ & $1.61 \mathrm{p} / \mathrm{mm}$ \\
\hline Mean & $1.61 \mathrm{p} / \mathrm{mm}$ & $1.61 \mathrm{p} / \mathrm{mm}$ & $1.61 \mathrm{p} / \mathrm{mm}$ & $1.61 \mathrm{p} / \mathrm{mm}$
\end{tabular}




\subsection{Linearity}

The darker side in the step wedge image was measured as level 1 at an SID of $130 \mathrm{~cm}$. As shown in Table 8, at an SID of $130 \mathrm{~cm}$, the mean SI value of image generated by S company showed an increase from 1,129.9 at level 1 to $1,773.6$ at level 11 . The mean SI value of image generated by D company showed an increase from 130.6 at level 1 to 947.5 at level 11 . However, at SID $180 \mathrm{~cm}$, the mean SI value of S company increased from $1,190.7$ at level 1 to $11,829.2$ at level 11. The mean SI value of D company increased from 178 at level 1 to 978.9 at level 11.

Table 8. SI of Step wedge at two SID levels $(130 \mathrm{~cm}$ and $180 \mathrm{~cm})$.

\begin{tabular}{|c|c|c|c|c|}
\hline \multirow{2}{*}{ manufacturer } & \multirow{2}{*}{ Step } & \multirow{2}{*}{ Area } & $130 \mathrm{~cm}$ & $180 \mathrm{~cm}$ \\
\hline & & & Mean \pm SD & Mean \pm SD \\
\hline \multirow{11}{*}{ S company } & 1 & 153 & $1,190.7 \pm 47.9$ & $1,190.7 \pm 47.9$ \\
\hline & 2 & 153 & $1,255.5 \pm 44.7$ & $1,255.5 \pm 44.7$ \\
\hline & 3 & 153 & $1,323.0 \pm 46.4$ & $1,323.0 \pm 46.4$ \\
\hline & 4 & 153 & $1,387.3 \pm 47.8$ & $1,387.3 \pm 47.8$ \\
\hline & 5 & 153 & $1,463.9 \pm 48.5$ & $1,463.9 \pm 48.5$ \\
\hline & 6 & 153 & $1,503.0 \pm 52.8$ & $1,503.0 \pm 52.8$ \\
\hline & 7 & 153 & $1,566.1 \pm 54.8$ & $1,566.1 \pm 54.8$ \\
\hline & 8 & 153 & $1,622.8 \pm 55.5$ & $1,622.8 \pm 55.5$ \\
\hline & 9 & 153 & $1,685.5 \pm 60.3$ & $1,685.5 \pm 60.3$ \\
\hline & 10 & 153 & $1,742.6 \pm 61.1$ & $1,742.6 \pm 61.1$ \\
\hline & 11 & 153 & $1,829.2 \pm 63.4$ & $1,829.2 \pm 63.4$ \\
\hline \multirow{11}{*}{ D company } & 1 & 153 & $178.0 \pm 29.5$ & $178.0 \pm 29.5$ \\
\hline & 2 & 153 & $257.4 \pm 37.0$ & $257.4 \pm 37.0$ \\
\hline & 3 & 153 & $325.6 \pm 44.1$ & $325.6 \pm 44.1$ \\
\hline & 4 & 153 & $404.5 \pm 50.1$ & $404.5 \pm 50.1$ \\
\hline & 5 & 153 & $488.3 \pm 55.6$ & $488.3 \pm 55.6$ \\
\hline & 6 & 153 & $565.9 \pm 60.4$ & $565.9 \pm 60.4$ \\
\hline & 7 & 153 & $644.1 \pm 61.7$ & $644.1 \pm 61.7$ \\
\hline & 8 & 153 & $730.1 \pm 64.3$ & $730.1 \pm 64.3$ \\
\hline & 9 & 153 & $812.0 \pm 68.1$ & $812.0 \pm 68.1$ \\
\hline & 10 & 153 & $907.8 \pm 71.3$ & $907.8 \pm 71.3$ \\
\hline & 11 & 153 & $978.9 \pm 68.7$ & $978.9 \pm 68.7$ \\
\hline
\end{tabular}

\section{Discussion}

Korea has systematically implemented quality control measures for special medical equipment under the laws and regulations since 2005. This quality control is reflected in the image quality of special medical equipment, efficiency of equipment use, reduced exposure, and reduced national health expenditure[5].The long-term use of diagnostic X-ray generators leads to deterioration in performance and quality of X-ray images. A factual survey of the image quality of CT, MRI and mammography units at domestic hospitals and clinics since July 2000 underscored the need for urgent quality control of image based on monitoring and inspection results showing inappropriate diagnostic image quality. The limitations should be addressed by qualified maintenance technicians, such as radiologists, radiation technologists, and medical physicists as a preventive measure [6].As of 2019, 19,500 diagnostic X-ray generators have been installed and operated in Korea.The management of tertiary and general hospitals is satisfactory; however, a few diagnostic X-ray generators were installed and operated in hospitals. Clinics and convalescent hospitals are required to implement safety management and quality control of images at a serious level of deterioration.

In Korea, despite electrical and mechanical safety regulations for diagnostic X-ray generators, there is no quality control of medical images due to the absence of legal or institutional regulations, and poor awareness of quality 
control measures for diagnostic X-ray generators. Therefore, legal regulations for the quality control of medical images should be established as a first step, and phantoms should be developed and used for quality control. However, significantly few medical and educational institutions carry expensive phantoms and implement quality control measures [7].Therefore, an evaluation index based on indigenous phantom for quality control was developed to measure field compliance, uniformity, step wedge, and low and high-contrast resolution in this study. The utility of the phantom was evaluated quantitatively and qualitatively using the diagnostic X-ray generator. Since the proposed SID of $130 \mathrm{~cm}$ minimized image enlargement and distortion in the DR system, the SID value of $130 \mathrm{~cm}$ combined with the existing SID value of $180 \mathrm{~cm}$ for long distance were used in this study [8].The field compliance of images generated by both $\mathrm{S}$ and $\mathrm{D}$ companies for quantitative evaluation was appropriate within $\pm 1 \%$. According to IEC 61223-2-11 [9] and the safety management rules for diagnostic radiation generators, the difference between radiation and light fields is $\pm 2 \%$. Accurate scanning and reduction in radiation exposure require appropriate adjustment of the field of radiation and light [10]. In the general X-ray examination, the exposure dose should be reduced by adjusting the field size to fit the patient region. The uniformity test based on IEC 61223-2-11 and previous studies represents an index that sets the ROI of the internal and external sides to measure and compare the regular changes in concentration throughout the images, which was adopted in this study [11]. In the experiment using Step wedge, both S and D companies verify the linearity of the DR system according to the SID change, and the changes in exposure dosage and differences in pixel values characterizing the equipment according to the height of the Step wedge were identified. The SI value of images produced by S company was high at similar optical density. For qualitative evaluation, the hole pattern was identified up to $0.8 \mathrm{~mm}$ under high-contrast resolution. The hole pattern was produced based on the image evaluation indices of CT among special medical equipment. For CT, the high-contrast resolution (spatial resolution) must be within $1.0 \mathrm{~mm}$, according to the Rules for the Installation and Operation of Special Medical Equipment, 2019. Bar pattern was produced by referring to the R-1W specifications of KSA 4902 (Resolution Chart for X-ray, 1979), and identified up to $1.6 \mathrm{LP} / \mathrm{mm}$ in this study. For the low-contrast resolution, a subjective C-D pattern was used to facilitate the evaluation of MR and CT image quality. The quality evaluation indices of the diagnostic X-ray generator were based on the C-D pattern. At SID $130 \mathrm{~cm}$, the image generated by S company was evaluated with 124.6 points, and that of D company with 116 points, and at SID $180 \mathrm{~cm}$, the image generated by S company scored 111.4 and that of D company 104.6 points.Occasionally, it is difficult to evaluate whether or not the quality of X-ray images is satisfactory based on regular inspection using a diagnostic $\mathrm{X}$-ray generator. Since the X-ray image is finally judged based on visual perception, a qualitative evaluation including the observer's area is also required. Therefore, the complex phantom that can measure and evaluate in parallel with quantitative and qualitative evaluation is needed.The study is limited by the inability to implement a hole pattern of less than $0.6 \mathrm{~mm}$ and a bar pattern greater than $2.0 \mathrm{LP} / \mathrm{mm}$ using the indigenous Phantom. To implement a hole pattern of less than $0.3 \mathrm{~mm}$ and a bar pattern of more than $4.0 \mathrm{LP} / \mathrm{mm}$, the production methods and materials need to be refined, along with design improvement depending on the characteristic X-rays emitted from the dotted circle. Besides, the advantages of X-ray generators using phantoms should be evaluated by verifying various tools according to performance differences depending on the years of use, equipment manufacturer, and hospital size.

\section{Conclusion}

Appropriate management and efficient use of diagnostic X-ray generators by qualified personnel is essential for quality control. A phantom for quality control of X-ray imaging was generated to quantitatively and qualitatively evaluate its utility in this study. The evaluation indices were analyzed by acquiring X-ray images at SIDs $130 \mathrm{~cm}$ and $180 \mathrm{~cm}$ of the diagnostic X-ray generators manufactured by S and D companies. In the quantitative evaluation, field compliance, uniformity, and linearity were verified. High-contrast and low-contrast resolution were evaluated qualitatively.In this study, the homegrown phantom was used to evaluate several indices concurrently, enabling quality control of X-ray images. The quality control used for quantitative and qualitative evaluation is believed to be accurate and yielded excellent outcomes.

\section{References}

1. Lee I. J., Kim Y. H., Kim C. N., Lee C. Y., Park K. Y. (2009). Evaluation of Image According to Exposure Conditions using Contrast-Detail Phantom for Chest Digital Radiography. Journal of radiological science and technology, 32(1), $25-32$.

2. Lee J. W. (2014). Implementation of a systematic radiation management program for reducing the patient dose on digital radiography examinations. Doctoral dissertation. Kwangwoon University. Seoul.

3. Jo G. H., Kang Y. H., Kim B. S. (2008). A Study on the Exposure Parameter and the Patient Dose for Digital Radiography System in Dae Goo. Journal of radiological science and technology, 31(2), 177 182. 
4. Shin S. I., Kim J. I., Kim S. C. (2008). The Study on the Reduction of Patient Surface Dose Through the use of Copper Filter in a Digital Chest Radiography. Journal of radiological science and technology, 31(3), $223-228$.

5. Kim N. S. (2011). A Study on the Awareness of Medical Imaging Quality Managers on the Quality Control of the Special Medical Equipments. Doctoral dissertation. Daegu Hanny University. Gyeongbuk Korea.

6. Choi J. I., Na D. K., Kim H. H., Shin Y. M., An K. J., \& Lee J. Y. (2004). Quality Control of Medical Imaging. Journal of the Korean Radiological Society), 50(5), 317-331.

7. Lee Y. K. (2019). Evaluation of the use of brain phantom made in $3 D$ printer in the field of nuclear medicine. Doctoral dissertation. Gachon University. Incheon.

8. Lim C. H., Park Y.W., Jung H. R., Yang O. N., Baek C. M. (2015). Appropriate inspection distance of digital X-ray imaging equipment for diagnosis International Journal of Applied Engineering Research, 8 (S8), 380-6.

9. IEC 61223-2-11. (2005). Evaluation and Constancy Testing in medical imaging Department-Part 2-11; Constancy tests- Equipment for General Direct Radiography.

10. Knag S. S., Go. S. K., Kim J. M. (2000). Radiation equipment quality control and experiment, 50-52. Jungmunkag publisher. http://www.jungmunkag.co.kr/

11. Jung S. H. (2016). Constancy Evaluation of Digital Radiography for Quality Assessment Standardization. Doctoral dissertation. Hanseo University. Chungcheongnam-do. 